\title{
Changes in the hepatic differentiation potential of human mesenchymal stem cells aged in vitro
}

\author{
Sang Luo^, Shuai Xiao, Yang Ai, Ben Wang, Yefu Wang \\ State Key Laboratory of Virology, School of Life Sciences, Wuhan University, Wuhan, China \\ Contributions: (I) Conception and design: S Luo; (II) Administrative support: Y Wang; (III) Provision of study materials or patients: S Xiao; (IV) \\ Collection and assembly of data: S Luo; (V) Data analysis and interpretation: S Luo, Y Ai, S Xiao, B Wang; (VI) Manuscript writing: All authors; (VII) \\ Final approval of manuscript: All authors. \\ Correspondence to: Yefu Wang. State Key Laboratory of Virology, School of Life Sciences, Wuhan University, Wuhan, China. \\ Email: wangyefu@whu.edu.cn.
}

Background: Due to their multipotency and ability for self-renewal, human umbilical cord-derived mesenchymal stem cells (hUC-MSCs) hold great promise for generating hepatocytes. Previous research has successfully generated hepatocytes from early-passage [i.e., passage (P)3] hUC-MSCs; however, the populations of early-passage cells are limited, and these cells cannot produce sufficient functional hepatocytes for large-scale application in clinical therapy. Thus, a thorough investigation of the hepatic differentiation potential of in vitro-aged hUC-MSCs is needed.

Methods: hUC-MSCs were passaged in vitro and subcultured every 3 days up to P8, and their morphology, proliferative capacity, liver-specific marker expression, and liver function at the end of each passage were analyzed. The efficiency of the hepatogenic differentiation of hUC-MSCs driven by a functional hit 1 (FH1)-based strategy at different passages was also evaluated.

Results: The in vitro-aged hUC-MSCs gradually displayed morphological inhomogeneity, had reduced proliferative capability, and exhibited senescent properties while maintaining adipogenic and osteogenic differentiation potential. Additionally, senescence also decreased the expression of messenger RNA (mRNA) levels in albumin (ALB) and alpha 1-antitrpsin (A1AT) in these cells and their relative protein expression, which is the marker of a mature hepatocyte. The liver function of the in vitro-aged hUC-MSCs also deteriorated gradually. Finally, the percentage of hepatocyte-like cells (HLCs) generated from in vitro-aged hUC-MSCs reduced significantly, and the mature hepatocyte functions, such as ALB secretion, glycogen synthesis, low-density lipoprotein (LDL) intake, and indocyanine green (ICG) uptake, also changed.

Conclusions: hUC-MSCs possess mature hepatocytes' specific markers and functions, which change gradually as they undergo cell senescence. Due to the loss of these properties within in vitro subcultures, the hepatic differentiation efficiency of in vitro-aged hUC-MSCs decreased dramatically in the late passage (P8). The current study provides valuable information can inform future research on liver disease.

Keywords: Human umbilical cord-derived mesenchymal stem cells (hUC-MSCs); cell aging; hepatic differentiation

Submitted Aug 31, 2021. Accepted for publication Oct 13, 2021.

doi: $10.21037 /$ atm-21-4918

View this article at: https://dx.doi.org/10.21037/atm-21-4918

^ ORCID: 0000-0003-2291-4295. 


\section{Introduction}

Liver failure is a serious disease that adversely impacts the patient's quality of life. About 2 million people die of liver failure every year, accounting for $3.5 \%$ of deaths worldwide (1). Orthotopic liver transplantation is the most effective treatment for liver failure. However, fewer than $10 \%$ of patients successfully receive liver transplantation due to a lack of donors, and most patients die while waiting for transplantation (2). The emergence of artificial livers has provided an effective treatment for patients with liver failure and affords those awaiting liver transplantation more time to find an appropriate liver source $(3,4)$. Primary hepatocytes are ideal cell models for bioengineered livers. However, due to the long duration of in vitro cultures, primary hepatocytes can easily lose their hepatocytic functions, which greatly limits their application in bioartificial livers. Thus, new cell sources urgently need to be found to replace primary hepatocytes (5).

Human stem cells are a promising candidate for primary human hepatocytes and regenerative medicine due to their ability for self-renewal and multipotency (6). Mesenchymal stem cells (MSCs) are a type of adult stem cell that originate from the mesoderm, which can express the surface antigens of CD73, CD90, and CD105, but not express the hematopoietic cell mareker CD34. They can selfrenew and have various differentiation potentials, including osteogenesis, adipogenesis, and chondrogenesis (7). In vivo and in vitro research has confirmed that MSCs have low immunogenicity and can promote the repair of damaged tissues (8). More importantly, as there are no ethical limitations, they are widely used in liver diseaserelated cell therapy. Since Friedenstein first discovered MSCs in 1970 (9), scientists have isolated these cells from a variety of tissues, including adipose tissues $(10,11)$, skeletal muscle (12), the umbilical cord (13), and the pancreas (14). Thus, hepatocyte-like cell (HLC) differentiation in human MSCs has the potential to overcome the limitations of primary hepatocytes.

However, MSCs are extremely sparse in primary tissues and account for only $0.01-0.001 \%$ of the cells in bone marrow (15). Freshly isolated MSCs are exceedingly rare and lack high homogeneity, and thus extensive in vitro expansion is required before they can be used in clinical practice and scientific research. Cell senescence is often accompanied by an increase in the number of expansions in vitro and potentially affects the characteristics and functionality of human umbilical cord-derived MSCs
(hUC-MSCs). Cell aging does not change the phenotype of hUC-MSCs; however, the repair capacity of aging cells in damaged tissues decreases significantly (16). Thus, to obtain more functional hepatocytes, the effect of cell aging caused by in vitro expansion on hepatic differentiation of hUCMSC must be considered.

Several studies have been conducted on human MSC aging; however, these studies have focused on changes in cell phenotype and the ability of osteogenesis, adipogenesis, and chondrogenesis $(17,18)$. As it relates to in vitro aging, the number of cell population doubling (NCPD) is considered as the actual age of the cultured cells. And the passaged MSCs showed a tendency to lose the ability of mitotic division during in vitro culture as evidenced by the decrease in NCPD by $69 \%$ from P3 to P8. However, there is no significant difference from passage $(\mathrm{P}) 4$ to $\mathrm{P} 8$ MSCs in the surface phenotype. Additionally, aged MSCs demonstrate adipogenic and osteogenic differentiation abilities (19). In a previous study, we found that MSCs exert certain liver functions, such as storing periodic acid-Schiff (PAS) and the uptake of low-density lipoprotein (LDL). To date, no research appears to have been conducted concerning whether cell senescence deteriorates the liver characteristics of undifferentiated MSCs or on the hepatogenic differentiation capacity of MSCs at different passages.

This study first identified the liver-specific markers expressed in undifferentiated hUC-MSCs from early passage $(\mathrm{P} 4)$ to late passage $(\mathrm{P} 8)$ in vitro and liver function. Then, to enable more hepatocytes to be acquired from limited cell resources, we further studied the effects of cell aging on the hepatogenic differentiation of in vitro-aged hUC-MSCs. We present the following article in accordance with the MDAR reporting checklist (available at https:// dx.doi.org/10.21037/atm-21-4918).

\section{Methods}

\section{bUC-MSC cultures}

hUC-MSCs at P2 (RC02003, Nuwacell Biotechnologies Co., Ltd., Hefei, China) were cultured in a serum-free human MSC (SF hMSC) culture medium (RP020101, Nuwacell ncMission hMSC Basal Medium, Nuwacell Biotechnologies Co., Ltd.) and supplement (RP02010-2. Nuwacell ncMission Supplement, Nuwacell Biotechnologies Co., Ltd.) at $37^{\circ} \mathrm{C}$ in a humidified atmosphere in a $5 \%$ carbon dioxide $\left(\mathrm{CO}_{2}\right)$ incubator. 


\section{Hepatocyte differentiation in vitro}

hUC-MSCs at P4-P8 were seeded in 6-well plates coated with $0.1 \%$ gelatin at a density of $1.5-3 \times 10^{4} / \mathrm{cm}^{2}$, and cells were cultured in an expansion medium at $37^{\circ} \mathrm{C}$ with $5 \% \mathrm{CO}_{2}$. Hepatogenic differentiation was performed as described previously (20). Briefly, when the cells' confluency level reached $100 \%$, the expansion medium was changed to $1 \mu \mathrm{M}$ of all-trans retinoic acid (ATRA; Abmole Bioscience, Houston, TX, USA) in Iscove's Modified Dulbecco's Medium (IMDM; Gibco, MD, USA) without serum. After 24 hours, the pretreatment medium was changed to IMDM with $100 \mathrm{nM}$ of IDE1, $3 \mu \mathrm{M}$ of CHIR99021, and $10 \mu \mathrm{M}$ of LY294002 (Abmole Bioscience). Next, the cells were incubated in serum-free IMDM supplemented with $100 \mathrm{nM}$ of IDE1, $10 \mu \mathrm{M}$ of LY294002, $250 \mathrm{nM}$ of LDN193189 (Abmole Bioscience), and $20 \mathrm{ng} / \mathrm{mL}$ of basic fibroblast growth factor (bFGF; PeproTech, Rocky Hill, NJ, USA) for 48 hours, and then changed to serum-free IMDM along with $100 \mathrm{nM}$ of IDE1, $10 \mu \mathrm{M}$ of LY294002, and $20 \mathrm{ng} / \mathrm{mL}$ of bFGF for another 24 hours. For the hepatocyte maturation stage, the differentiation medium was composed of Hepatocyte Basal Medium (HBM, Lonza, Basal, Switzerland) supplemented with $15 \mu \mathrm{M}$ of functional hit 1 (FH1; Abmole Bioscience), $20 \mathrm{ng} / \mathrm{mL}$ of bFGF, $30 \mathrm{ng} / \mathrm{mL}$ of onscostatin M (OSM; PeproTech), $2 \times 10^{-5} \mathrm{~mol} / \mathrm{L}$ of dexamethasone (Dex; Abmole), and $1 \%$ insulin-transferrin-selenium supplement-X (ITS-X; SigmaAldrich, St. Louis, MO, USA). In the final step, the medium was changed every 2 days.

\section{bUC-MSC adipogenic and osteogenic differentiation}

hUC-MSCs at P4 and P8 were cultured in SF hMSC culture medium; when the cell confluence reached $85 \%$, the culture medium was changed to adipogenic differentiation medium (Nuwacell Adipogenic Differentiation Kit; Nuwacell Biotechnologies Co., Ltd.) or osteogenic differentiation medium (Nuwacell Osteogenic Differentiation Kit, Nuwacell Biotechnologies Co., Ltd.), and the medium was changed every 3 days. At 21 days, the cells were assessed for extracellular matrix synthesis.

\section{Histology}

The cells collected in the differentiation experiments were fixed in 4\% paraformaldehyde for 30 minutes at room temperature and washed with phosphate-buffered saline
(PBS) twice. The adipogenic samples were incubated with Oil Red O (Nuwacell Adipogenic Differentiation Kit; Nuwacell Biotechnologies Co., Ltd.) working reagent and left for 1 hour in a dark room to stain the lipids. To visualize the calcium, the osteogenic samples were incubated with 2\% Alizarin Red S (Nuwacell Osteogenic Differentiation Kit, Nuwacell Biotechnologies Co., Ltd.) dissolved in deionized water for 1 hour. The sample was then rinsed 5 times with PBS. Color images were visualized under a light microscope.

\section{Flow cytometry}

At each passage, the hUC-MSCs were seeded in 6-well plates. When the cell confluence reached $60 \%$, the culture medium was changed to a 5-ethynyl-2'-deoxyuridine (EdU; Beyotime Biotechlogogy, Shanghai, China) working reagent that was diluted with a culture medium (with a final concentration of $20 \mu \mathrm{M}$ ) and left for 1 hour. The cells were then harvested and fixed with $4 \%$ paraformaldehyde for 15 minutes at room temperature and rinsed with PBS for 3 minutes. Cells were then blocked with $0.2 \%$ Triton X-100 (prepared with PBS) for 15 minutes at room temperature. After this, $0.5 \mathrm{~mL}$ of click reaction reagent was added to each sample, which was then left for 30 minutes in a dark room. After the cells were rinsed 3 times with PBS, their proliferation ability was analyzed with flow cytometry.

\section{RNA isolation and quantitative real-time polymerase chain reaction ( $q R T-P C R)$}

Total RNA was isolated from the hUC-MSCs, definitive endoderm (DE) cells, hepatic progenitor cells, and iHeps using the MiniBEST Universal RNA Extraction Kit (Takara Bio, Tokyo, Japan) in accordance with the manufacturer's instructions. RNA was then reverse transcribed into complementary DNA (cDNA) using the PrimeScript RT Reagent Kit with gDNA Eraser (Perfect Real Time; Takara Bio). The cDNA was then amplified by fluorescent $\mathrm{qRT}$ PCR. The expression of liver specific genes (Table 1) was assessed using an ABI Prism 7500 Sequence Detection System (Applied Biosystems, Carlsbad, CA, USA) and the TB Green Premix Ex Taq II (Takara Bio). Glyceraldehyde 3-phosphate dehydrogenase (GAPDH) expression was used as the internal standard. The data from 3 independent experiments were collected. 
Table 1 Primers used for qRT-PCR in detecting liver-specific gene expression

\begin{tabular}{ll}
\hline Gene & Primer sequence (5' to $3^{\prime}$ ) \\
\hline GAPDH & F: ACCATCTTCCAGGAGCGAGAT \\
R: ATGACGAACATGGGGGCATC & F: GCACAGAATCCTTGGTGAACAG \\
R: ATGGAAGGTGAATGTTCAGCA & F: AGGTGCCTATGATGAAGCGT \\
A1AT & R: TGGCAGACCTTCTGTCTTCATT \\
& F: TCCAGCGACCTTCCTCATCCAC \\
p21 & R: TCCATAGCCTCTACTGCCACCATC \\
& F: GCCCATCCTCACCATCATCACAC \\
& R: GCACAAACACGCACCTCAAAGC \\
& F: GGCACCAGAGGCAGTAACCATG \\
& R: CTAAGTTTCCCGAGGTTTCTCAGAGC
\end{tabular}

qRT-PCR, quantitative real-time polymerase chain reaction; GAPDH, glyceraldehyde 3-phosphate dehydrogenase; ALB, albumin; A1AT, alpha 1-antitrpsin.

\section{Immunofluorescence staining experiment}

The cells were fixed with $4 \%$ paraformaldehyde for 15 minutes and blocked with PBS containing $0.5 \%$ Triton $\mathrm{X}-100$ for 20 minutes at room temperature. After being washed with PBS 3 times, cells were blocked with normal goat serum at room temperature for 30 minutes and were then were incubated with primary antibodies at $4{ }^{\circ} \mathrm{C}$ overnight. On the second day, cells were washed with PBS 3 times and then incubated with fluorescent secondary antibody at the appropriate concentration at $37^{\circ} \mathrm{C}$ for 1 hour. 4',6-diamidino-2-phenylindole (DAPI) was used to stain the cell nuclei. Image acquisition and processing were performed using a fluorescence microscope (Olympus, Tokyo, Japan).

The positive cells were counted using IPP6.0 software (Media Cybernetics, Rockville, MD, USA). The primary antibody used in the immunofluorescence staining experiments were as follows: rabbit anti-human forkhead box A2 (FoxA2; 1:200; Proteintech, Wuhan, China; 224741-AP), mouse anti-human sex determining region $\mathrm{Y}$ (SRY)-box17 (SOX17; 1:200; R\&D Systems, MN, USA; MAB1924-SP), rabbit anti-human $\alpha$-fetoprotein (AFP; 1:100; Santa Cruz Biotechnology, Dallas, TX, USA; sc8399), mouse anti-human hepatocyte nuclear factor $4 \alpha$ (HNF4a; 1:500; Santa Cruz; sc-374229), rabbit anti-human albumin (ALB; 1:200; Proteintech; 16475-1-AP), mouse anti-human alpha 1-antitrpsin (A1AT; 1:100; Proteintech; 66135-1-Ig). The secondary antibody used in immune fluorescence staining experiments were as follows: goat antirabbit immunoglobin G (IgG; 1:100; Boster Bio, Wuhan, China; BA1032) and goat anti-mouse IgG (1:100; Boster; BA1101).

\section{Western blot}

The cells were dissolved in RIPA cell buffer (Beijing Solarbio Science \& Technology Co., Ltd., Beijing, China), and the proteins were separated via $10 \%$ sodium dodecyl sulfatepolyacrylamide gel electrophoresis (SDS-PAGE) gel, which was followed by electrotransferred onto nitrocellulose membranes (EMS; Millipore, Burlington, MA, USA). The membrane was saturated with PBS containing 5\% skim fat milk and $0.1 \%$ Tween 20 (PBST) for 1 hour at room temperature and incubated with the following primary antibodies: rabbit anti-human ALB (1:5,000; Proteintech; 16475-1-AP) and mouse anti-human A1AT (1:100; Proteintech; 66135-1-Ig) overnight at $4{ }^{\circ} \mathrm{C}$. Following washing in tris-buffered saline with Tween 20 , the membranes were incubated with goat anti-rabbit IgG- horseradish peroxidase HRP) secondary antibody $(1: 10,000$; Boster Bio; BA1051) or goat anti-mouse IgG-HRP secondary antibody (1:10,000; Boster Bio; BA1054) for 1 hour at room temperature. Human GAPDH was used as the control for protein loading.

\section{PAS staining for glycogen storage}

A PAS staining kit (Beijing Solarbio Science \& Technology Co., Ltd.) was used for the capacity of glycogen storage analysis. According to the manufacturer's instructions, the cells were fixed and oxidized for 15 minutes. After being washed with water and deionized water 2 and 3 times, respectively, the PAS reagent was added, and the samples were incubated in the dark at room temperature for 20 minutes. Subsequently, the samples were washed with flowing water for 2 minutes and Mayer's hematoxylin staining solution was added. The cells were observed under light microscopy.

\section{Indocyanine green (ICG) uptake assay}

To analyze the ICG uptake ability of cells, ICG (Abmole Bioscience) was dissolved in dimethyl sulfoxide (DMSO) 
for preparation of a $10 \mathrm{mg} / \mathrm{mL}$ stock solution. The stock solution was then freshly diluted in a culture medium and added to the cells at a final concentration of $1 \mathrm{mg} / \mathrm{mL}$. After being incubated for 30 minutes at $37^{\circ} \mathrm{C}$, cells were washed with PBS 3 times, and the cellular uptake of ICG was observed by light microscopy.

\section{Cellular uptake ability of LDL}

The cellular uptake ability of LDL was detected with a 1,1'-dioctadecyl-3,3,3',3'-tetramethylindocarbocyanine perchlorate (DiI)-Ac-LDL staining kit (Beijing Solarbio Science \& Technology Co., Ltd.). Briefly, the cells were incubated in culture medium containing $20 \mu \mathrm{g} / \mathrm{mL}$ DiIlabeled acetylated LDL (acLDL) for 4 hours at $37^{\circ} \mathrm{C}$, after which they were washed and visualized under fluorescence microscopy.

\section{Urea production analysis}

The cells from each differentiation group were cultured in IMDM with $10 \mathrm{mM} \mathrm{NH}_{4} \mathrm{Cl}$ for 24 hours. The supernatants were collected and evaluated using a commercial urea assay kit (Quantichrom Urea assay Kit, Bioassay Systems, Hayward, CA, USA).

\section{Statistical analysis}

Statistical analyses were performed using GraphPad Prism 6.0 (GraphPad Software, San Diego, CA, USA), the data from which are presented as mean \pm standard deviation (SD). The differences between the two groups were compared by Student's $t$-tests, and multiple groups were compared by one-way analysis of variance in conjunction with a Tukey's multiple comparisons test. A P value $<0.05$ was considered statistically significant.

\section{Results}

\section{Decreased proliferative capability and differentiation potential of in vitro-aged bUC-MSCs}

hUC-MSCs were subcultured every 3 days until cell confluence reached $85 \%$. At the end of each passage, the morphology of the cells was captured. Microscopic images demonstrated that the MSCs kept their normal spindle shape at the early passage up to P6 and showed less homogeneity at a later passage, characterized by an irregular, flatted geometry and enlarged size (Figure 1A). A reduction in proliferative capability accompanied the changes in morphology, and the positive rate of $\mathrm{EdU}$ in cultured cells dropped significantly from $98.33 \%$ at $\mathrm{P} 4$ to $11.8 \%$ at $\mathrm{P} 8$ (Figure $1 \mathrm{~B}, 1 \mathrm{C}$ ). Similarly, the messenger RNA (mRNA) expression level of the aging-related genes in P8-MSCs was dramatically upregulated compared to that in P4-MSCs (Figure 1D). Adipogenic and osteogenic differentiation capacities are another characterization of hUC-MSCs. However, hUC-MSCs at both P4 and P8 could differentiate into adipocyte-like cells and possessed osteogenic potential ability (Figure $1 E, 1 F$ ).

\section{Liver-specific marker expression level of bUC-MSCs affected by cell senescence}

To identify the liver-specific marker of hUC-MSCs, we first used immunofluorescence staining and western blot to investigate the protein expression of ALB and A1AT. There was a gradual decrease in the percentage of cells expressing A1AT, and the expression in the P4-MSCs was distinctly higher than that in the P8-MSCs; however, there were no significant differences in the expression of ALB among the various passages (Figure $2 A, 2 B$ ). The western blot analysis results were consistent with the immunofluorescence staining analysis (Figure 2C). Further, the liver-specific gene expression of ALB and A1AT for the in vitro-aged hUC-MSCs qualified by qRT-PCR was significantly higher in P4-MSCs than in P8-MSCs (Figure 2D). These results demonstrated that undifferentiated hUC-MSCs could express liver-specific genes and proteins, but those expression levels gradually decreased for in vitro-aged MSCs.

\section{Liver function of bUC-MSCs decreased by cell senescence}

The liver is an important metabolic center. It can store glycogen and uptake ICG and acLDL. PAS staining was used to assess the cytoplasmic glycogen storage granules at various passages of hUC-MSCs. The positive signal of PAS staining in aging hUC-MSCs gradually decreased (Figure 3A). In the LDL uptake experiments, the fluorescent probe DiI accumulated in the P8-MSCs was less than that accumulated in the P4-MSCs (Figure 3B). However, neither the early nor late passage hUC-MSCs possessed any metabolic capacity for ICG uptake (Figure 3C). Thus, in vitro-aged hUC-MSCs demonstrated a degree of mature hepatocyte function. 
A P4 P5
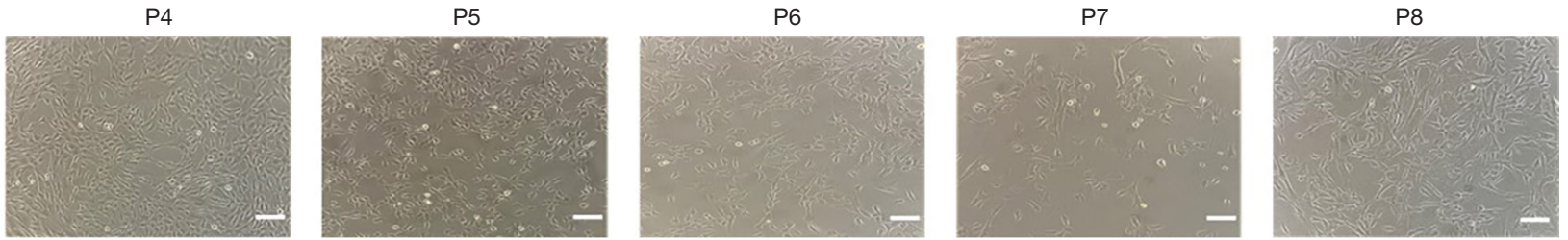

B

Tube4: P1
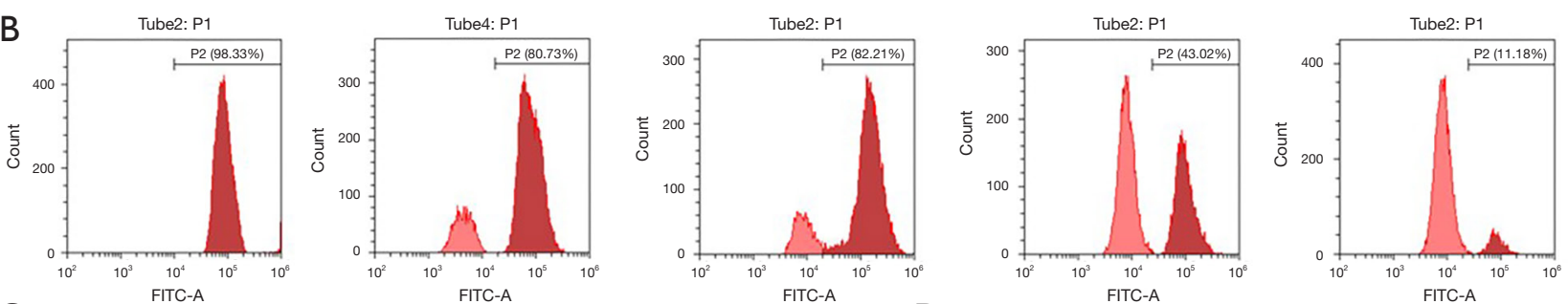

C

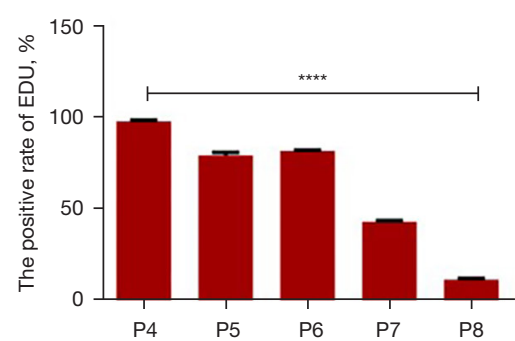

D

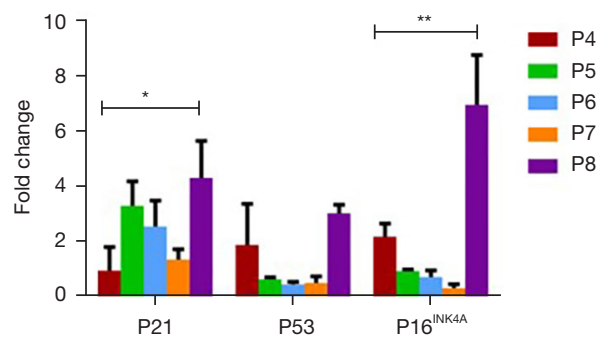

E
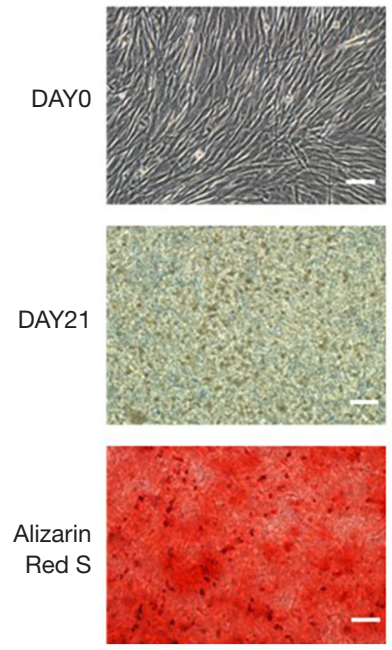

P8
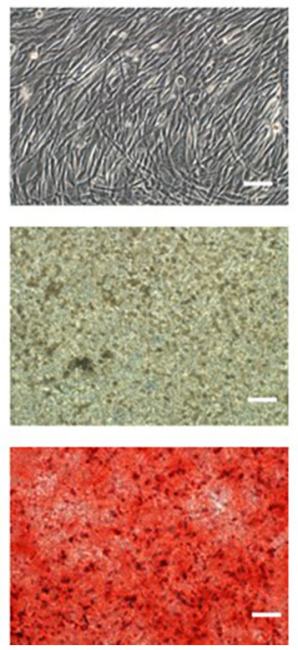

F

DAYO

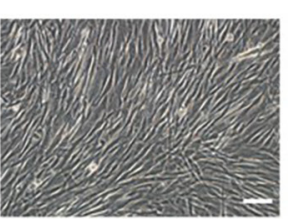

DAY21

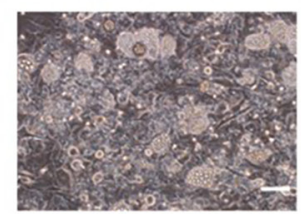

Oil

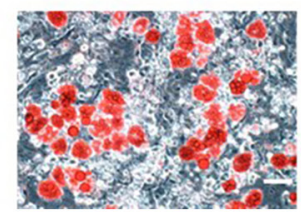

P8
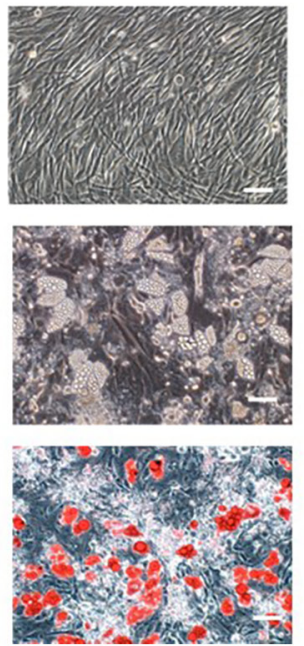

Figure 1 Identification of in vitro-aged hUC-MSCs. (A) Morphological representation of in vitro-aged hUC-MSCs. Scale bar $=100 \mu M$. (B) The proliferative capability of in vitro-aged hUC-MSCs according to EdU staining. (C) EdU-positive cell rate analysis of hUC-MSCs at various passages. (D) The mRNA expression level was determined by qRT-PCR. (E) Phase-contrast images of osteogenic samples were captured at designated time points. The cells were staining with 2\% Alzarin Red S for $60 \mathrm{~min}$. Scale bar =200 $\mu \mathrm{m}$. (F) Phase-contrast images of adipogenic samples were captured at designated time points. The cells were staining with Oil Red O for $60 \mathrm{~min}$. Scale bar =200 $\mu \mathrm{m}$. *, $\mathrm{P}<0.05 ;{ }^{* *}, \mathrm{P}<0.01 ;{ }^{* * *}, \mathrm{P}<0.0001$. The data are expressed as mean $\pm \mathrm{SD}(\mathrm{n}=3)$. hUC-MSCs, human umbilical cord-derived mesenchymal stem cells; P, passage; EdU, 5-ethynyl-2'-deoxyuridine; qRT-PCR, quantitative real-time polymerase chain reaction; mRNA, messenger RNA; SD, standard deviation. 

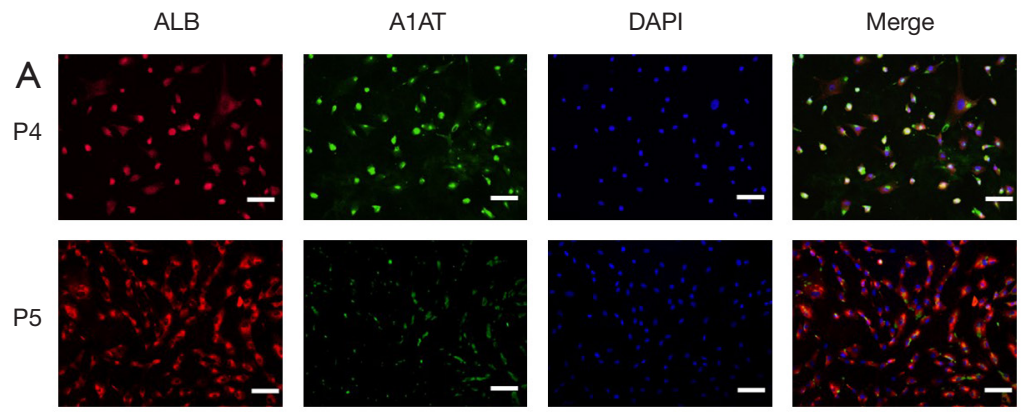

P6
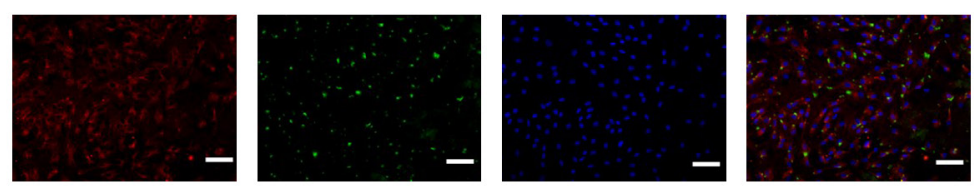

P7
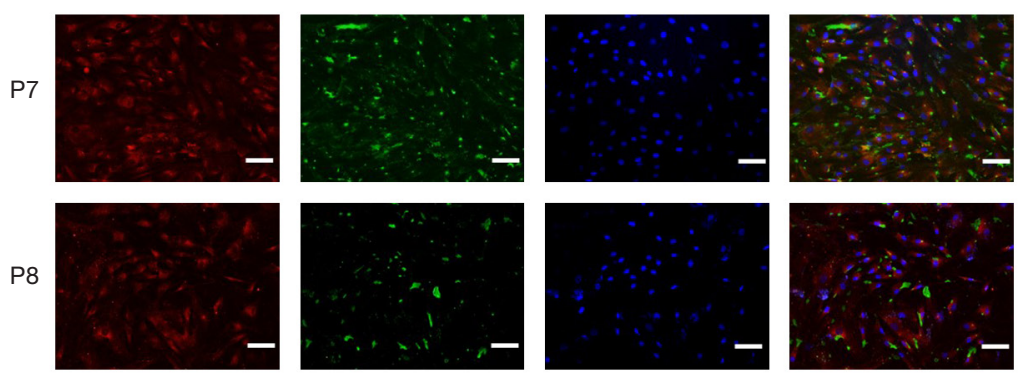

B
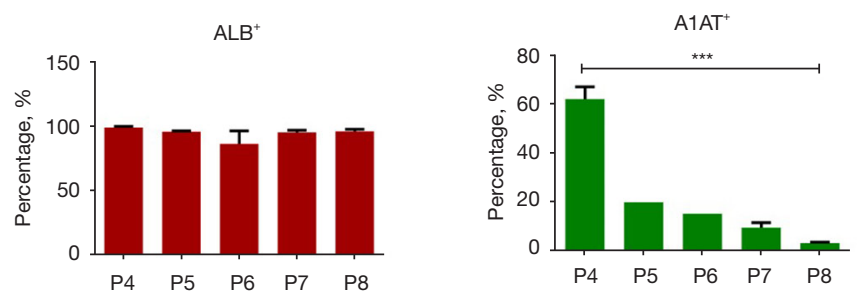

C
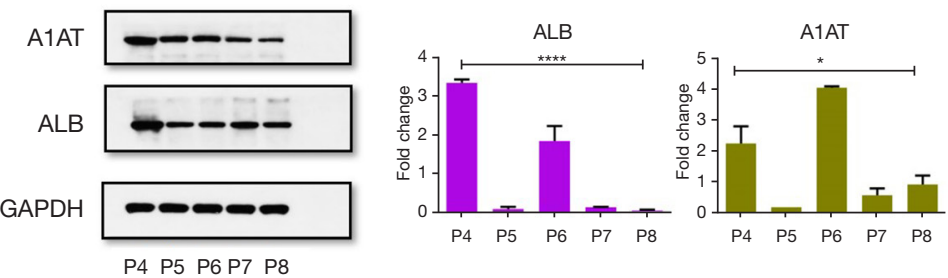

Figure 2 In vitro-aged hUC-MSCs expressing the liver-specific markers of ALB and A1AT. (A) Immunofluorescence of ALB and A1AT expression. Scale bar $=100 \mu$ M. (B) Immuno-positive cell ratio of the hUC-MSCs. (C) Western blot analysis of ALB and A1AT protein levels in P4-P8-hUC-MSCs. GAPDH was used as a control. (D) qRT-PCR analysis of liver-specific gene expression in undifferentiated hUCMSCs. *, $\mathrm{P}<0.05$; *** $\mathrm{P}<0.001$; ${ }^{* * *}, \mathrm{P}<0.0001$. The data are presented as mean $\pm \mathrm{SD}(\mathrm{n}=3)$. hUC-MSCs, human umbilical cord-derived mesenchymal stem cells; ALB, albumin; A1AT, alpha1 antitrypsin; P, passage; GAPDH, glyceraldehyde 3-phosphate dehydrogenase; qRTPCR, quantitative real-time polymerase chain reaction; SD, standard deviation. 
P4

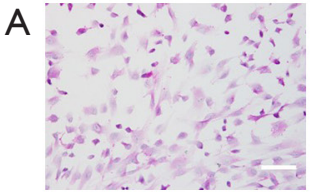

B

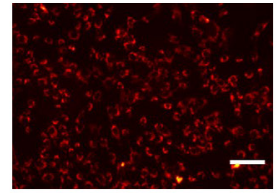

C

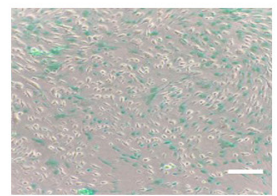

P5
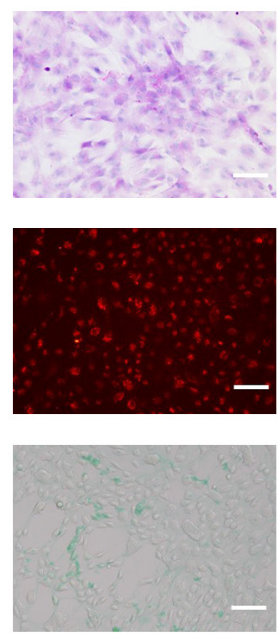

P6
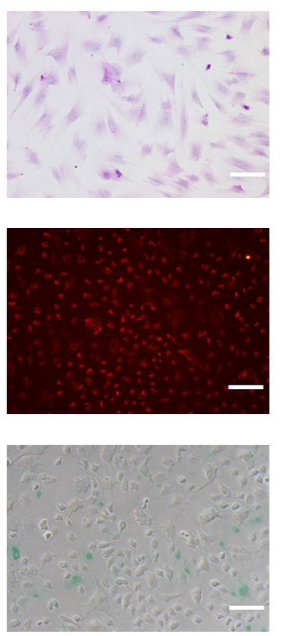

P7
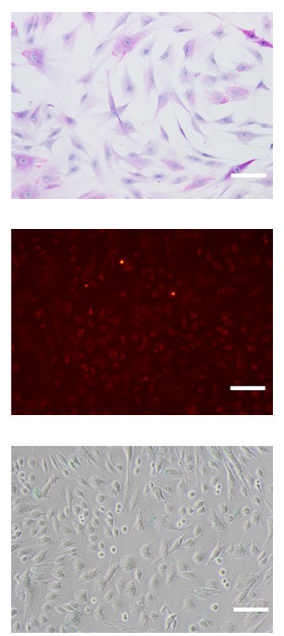

P8
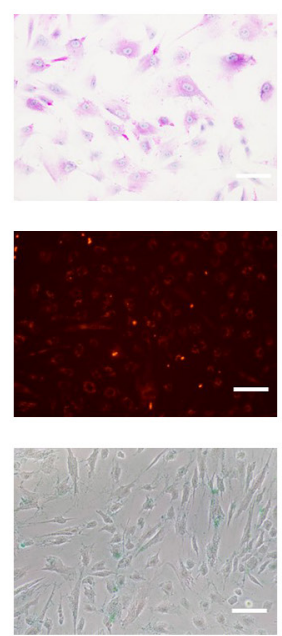

Figure 3 Undifferentiated hUC-MSCs with certain liver functions. (A) Glycogen storage analysis via PAS staining in undifferentiated hUC-MSCs. (B) Analysis of LDL uptake ability. (C) ICG uptake analysis. Scale bar $=100 \mu M$. hUC-MSCs, human umbilical cord-derived mesenchymal stem cells; PAS, periodic acid-Schiff; LDL, low-density lipoprotein; ICG, indocyanine green.

\section{Hepatic differentiation ability of bUC-MSCs affected by cell senescence}

The initial stage of hepatic differentiation involves inducing DE cell formation. Microscope images demonstrated that both early- and late-passage hUC-MSCs underwent significant changes in morphology, acquiring a short spindle shape at 2-day differentiation; however, the density of DE cells in P4-MSCs was much higher than that in P8-MSCs (Figure 4A). Immunofluorescent staining showed that the efficiency of DE cell formation decreased by $92.857 \%$ and $8.19 \%$ from $\mathrm{P} 4$ to $\mathrm{P} 8$, respectively (Figure $4 B, 4 C$ ), which was consistent with the changes in morphology.

Gradual changes in the shape of cells were observed when the DE cells differentiated from $\mathrm{P} 4$ to $\mathrm{P} 8 \mathrm{hUC}-$ MSCs during the second stage of differentiation. Specifically, the cells acquired a long spindle-like morphology at the endpoint of the hepatic progenitor cell differentiation stage (Figure $5 A$ ). Similarly, the expression of hepatic specific proteins (AFP and $\mathrm{HNF} 4 \alpha$ ) were used in the immunofluorescent staining, and the percentage of double-positive $\mathrm{AFP}^{+} \mathrm{HNF} 4 \alpha^{+}$ranged from $90.92 \%$ at $\mathrm{P} 4$ to $27.62 \%$ at $\mathrm{P} 8$ (Figure $5 B, 5 C$ ). The last stage of differentiation resulted in the generation of HLCs. The hepatic progenitor cells derived from P4-P8 hUCMSCs acquired a polygonal shape at the endpoint of the hepatocyte maturation stage; however, the cell morphology of the HLCs derived from the P8-hUC-MSCs was inconsistent with the HLCs derived from the early passage (P4-P7) of hUC-MSCs, and the cell density was extremely low compared to that in HLCs derived from early-passage hUC-MSCs (Figure 6A). Additionally, immunofluorescent staining showed that the strongest expression of $\mathrm{ALB}^{+} \mathrm{A} 1 \mathrm{AT}^{+}$was detected in the HLCs derived from P4MSCs. Conversely, there was only a 7.6\% expression of $\mathrm{ALB}^{+} \mathrm{A} 1 \mathrm{AT}^{+}$in the HLCs differentiated from P8-MSCs (Figure 6B,6C).

\section{The liver function of $H L C$ differentiated from $b U C$ - MSCs was decreased by cell senescence}

Glycogen synthesis is a biochemical function of mature hepatocytes in clinic. PAS staining results showed that hepatocytes differentiated from both early- and late-passage hUC-MSCs could store glycogen (Figure $7 A$ ). However, there were gradual changes in the glycogen staining color from dark red/purple to pink, demonstrating that HLCs differentiated from P4-MSCs had better preservation potential for storing glycogen (Figure $7 A$ ). Additionally, HLCs derived from P4-P7-hUC-MSCs took up ICG and acLDL; however, HLCs generated from the P8-hUCMSCs showed a limited capacity to do this (Figure $7 B, 7 C$ ). To assess the metabolic function of induced hUC-MSCs, 

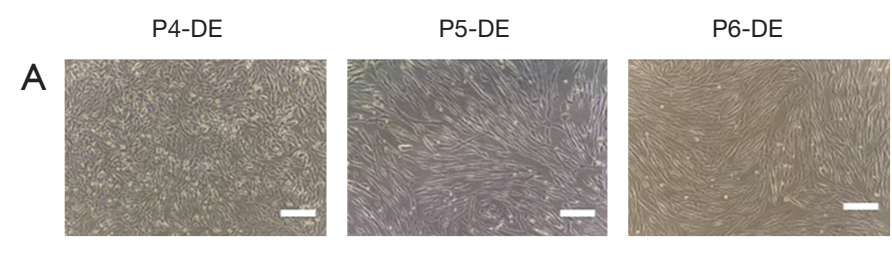

P7-DE
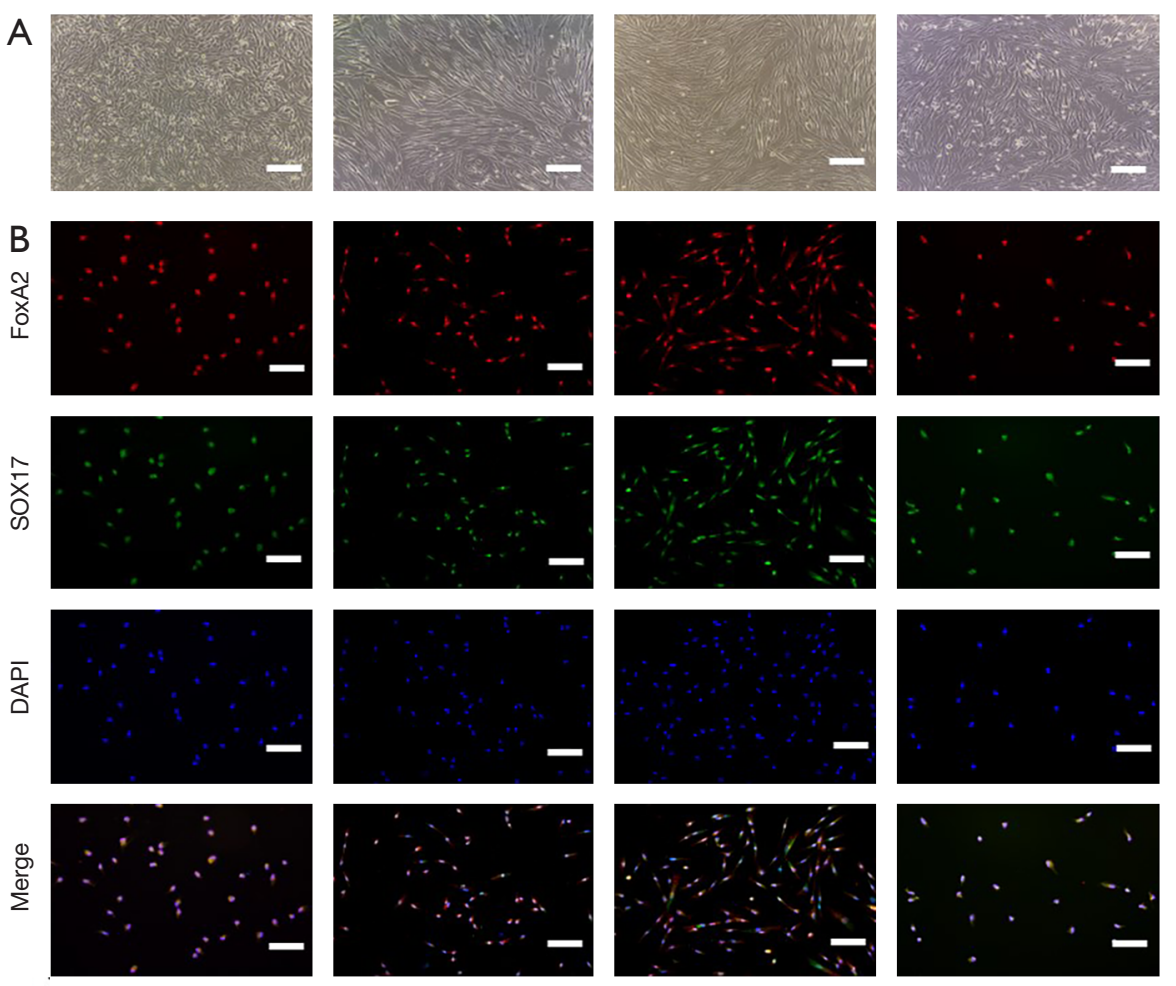

SOX $17^{+}$FoXA2 $2^{+}$

C

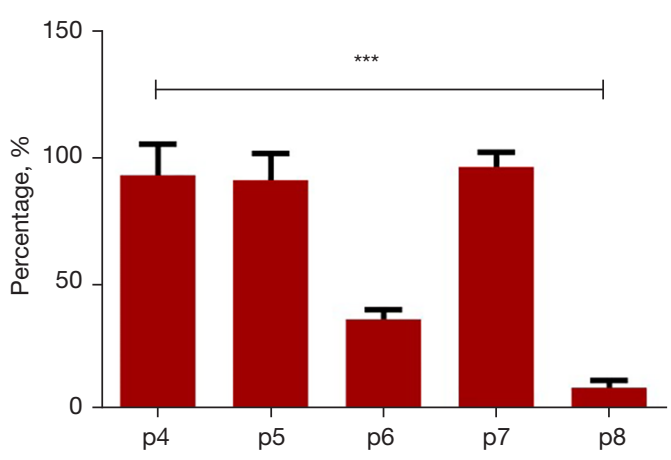

Figure 4 Identification of DE cells derived from the in vitro-aged hUC-MSCs. (A) Morphological representative of DE cells derived from in vitro-aged hUC-MSC-derived. Scale bar $=100 \mu \mathrm{M}$. (B) Immunofluorescence staining of the DE protein SOX17 and FoxA2 in DE cells derived from P4-P8-hUC-MSCs. Scale bar $=100 \mu M$. (C) Immuno-positive cell ratio of SOX17 $7^{+}$FoxA2 ${ }^{+}$in $\mathrm{DE}$ cells. ***, $\mathrm{P}<0.001$. The data are expressed as mean $\pm \mathrm{SD}(\mathrm{n}=3)$. DE, definitive endoderm; hUC-MSCs, human umbilical cord-derived mesenchymal stem cells; P, passage; SOX17, sex determining region Y-box17; FoxA2, forkhead box A2; SD, standard deviation; DAPI, 4',6-diamidino-2-phenylindole.

we examined urea secretion at the hepatocyte maturation stage of the differentiation process; however, there were no significant differences among HLCs derived from the hUC-MSCs at different passages (Figure 7D). Furthermore, western blot analysis was used to examine the secretion of hepatocyte-specific protein. The results showed that the levels of the proteins in the cells differentiated from the early-passage hUC-MSCs were higher than those in the 


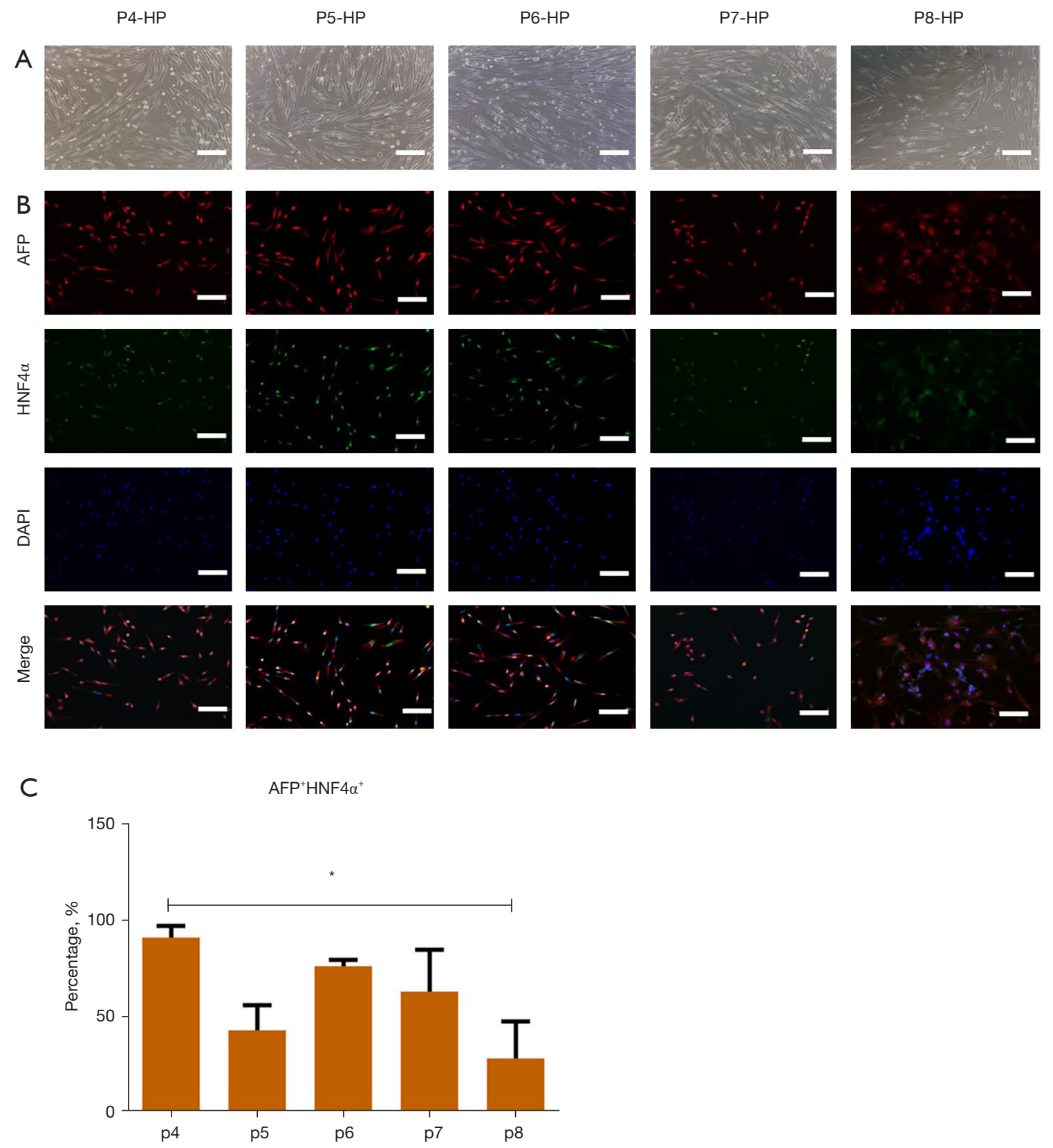

Figure 5 Changes of hepatic lineage markers expressed in hepatic progenitor cells derived from in vitro-aged hUC-MSCs. (A) Morphological changes at the hepatic progenitor cell differentiation stage. Scale bar $=100 \mu \mathrm{M}$. (B) Immunofluorescence staining of AFP and $\mathrm{HNF} 4 \alpha$ protein level expression in hepatic progenitor cells. Scale bar $=100 \mu \mathrm{M}$. (C) Immuno-positive cell ratio of $\mathrm{AFP}^{+} \mathrm{HNF} 4 \alpha^{+}$in the total differentiated cells. *, $\mathrm{P}<0.05$. The data are expressed as mean \pm SD ( $\mathrm{n}=3)$. hUC-MSCs, human umbilical cord-derived mesenchymal stem cells; AFP, $\alpha$-fetoprotein; HNF4 $\alpha$, hepatocyte nuclear factor 4 $\alpha$; SD, standard deviation; P, passage; DAPI, 4',6-diamidino-2-phenylindole. 

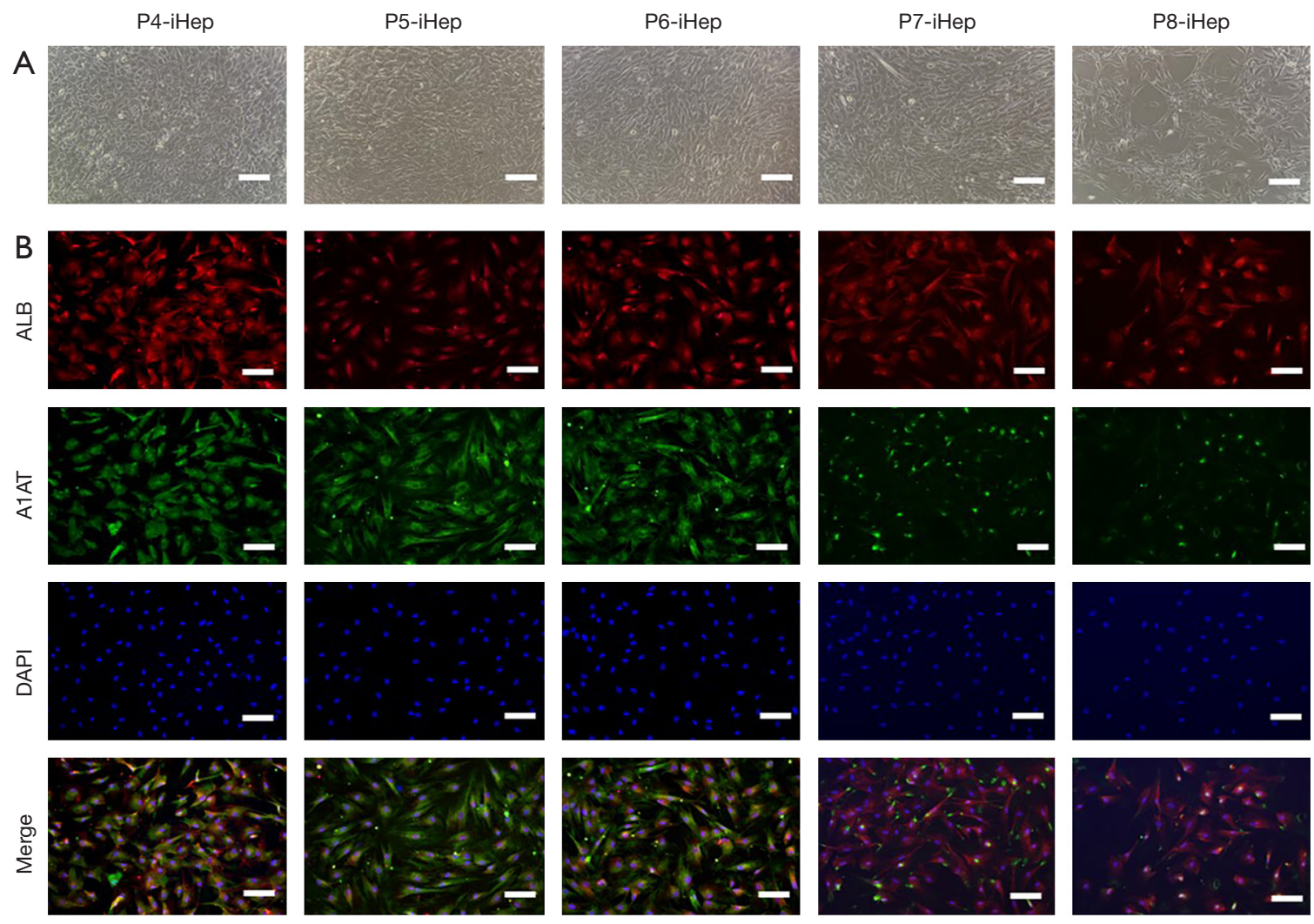

C $\mathrm{ALB}^{+} \mathrm{A} 1 \mathrm{AT}^{+}$

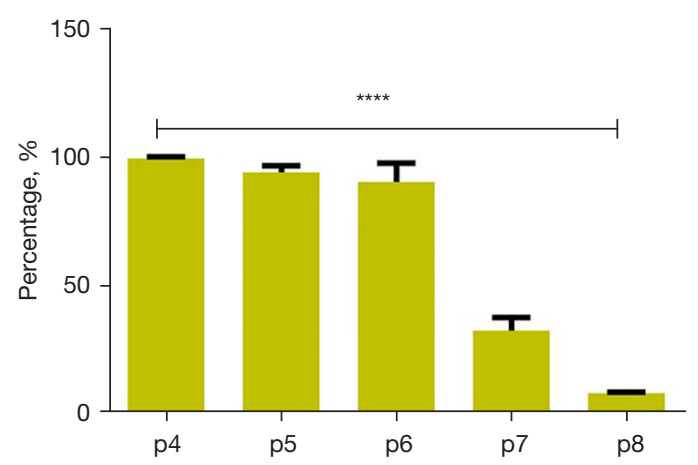

Figure 6 Identification of iHeps derived from the in vitro-aged hUC-MSCs. (A) Representative phase-contrast photographs of iHeps derived from P4-P8-hUC-MSCs. Scale bar $=100 \mu M$. (B) Immunofluorescence staining of the protein level of ALB and A1AT in the iHeps. Scale bar $=100 \mu M$. (C) Immuno-positive cell ratio of $\mathrm{ALB}^{+} \mathrm{AlAT}^{+}$in the total differentiated cells. ${ }^{* * * *}, \mathrm{P}<0.0001$. The data are expressed as mean $\pm \mathrm{SD}(\mathrm{n}=3)$. iHeps, induced hepatocyte-like cells; hUC-MSCs, human umbilical cord-derived mesenchymal stem cells; $\mathrm{P}$, passage; ALB, albumin; A1AT, alpha 1-antitrpsin; SD, standard deviation. 

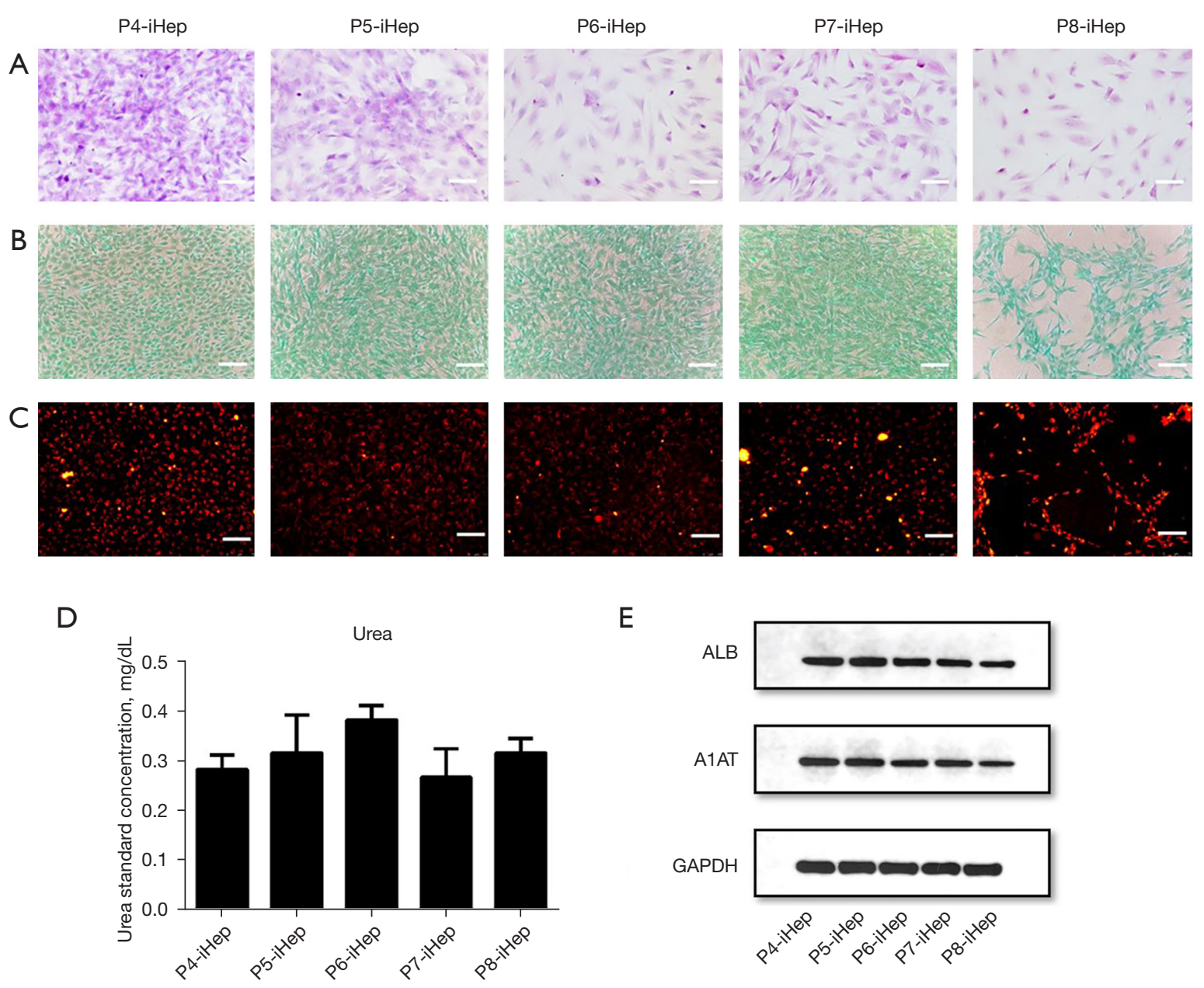

Figure 7 Characterization of iHeps derived from the in vitro-aged hUC-MSCs. (A) Glycogen storage analysis via PAS staining in iHeps derived from P4-P8-hUC-MSCs; (B) ICG intake; (C) LDL uptake. Scale bar $=100 \mu M$. (D) Urea production analysis. (E) Western blots analysis of hepatic markers in iHep. GAPDH was used as an internal control. The data are expressed as mean \pm SD ( $=3$ ). iHeps, induced hepatocyte-like cells; hUC-MSCs, human umbilical cord-derived mesenchymal stem cells; PAS, periodic acid-Schiff; P, passage; ICG, indocyanine green; LDL, low-density lipoprotein; GAPDH, glyceraldehyde 3-phosphate dehydrogenase; SD, standard deviation; ALB, albumin; A1AT, alpha 1-antitrpsin.

cells differentiated at late passages (P8; Figure 7E).

\section{Discussion}

There is a worldwide shortage of donor livers available for orthotropic liver transplants and hepatocyte transplantation therapies (21). In addition to their therapeutic potential, functional hepatocytes can facilitate the study of molecular and genetic aspects of human liver disease and provide a platform for drug toxicity screenings and the discovery of novel pharmaceuticals. They further hold considerable potential for treating a wide array of metabolic diseases (22). As the demand for human hepatocytes heavily outweighs their availability, finding new cell resources as substitutes for primary hepatocytes is imperative.

MSCs have the potential for self-renewal and immunosuppression and are attractive candidates for regenerative medicine (8). Hayflick et al. reported that MSCs stored at a low temperature could proliferate after 27 years (23). Several studies have applied MSCs to clinical cell therapy $(24,25)$. Compared with adult organ-derived MSCs, hUC -MSC can be obtained through non-invasive 
mehthods, it is also available for collection after delivery and remain viable after long-term cryopreservation. Most importantly, it has lower immunogenicity than other adult MSC tissue sources (26,27). However, MSCs are rarely in primary tissues and account for only $0.01-0.001 \%$ of the cell numbers in bone marrow (15), a figure much lower than that in the umbilical cord and peripheral blood (28). Due to their unique characteristics, isolated MSCs have a high degree of heterogeneity that cannot meet the standards of cell differentiation. Thus, in vitro expansion is needed to purify them. MSCs expand in vivo for several generations without losing the expression of specific differentiation phenotypes; however, cell senescence may deteriorate the ability of MSCs to adapt to the injured tissue and thus their ability to support the regeneration of tissue (15). The process of cell senescence was initially proposed by Hayflick (29), who believed that cell senescence is a process of cell life and development caused by a variety of factors, including tumor suppressor activation, chromatin change, apoptosis, and frequent protein synthesis. Aging cells still display metabolic activity, but their cell morphology and biological functions change significantly, including their proliferation ability and differentiation potential.

Cell replicative senescence is related to telomerase activity in the process of cell culture and expansion in vitro. Notably, telomere length shortens in the process of cell replication, which is responsible for the decrease of telomerase activity (30). Cell expansion can also be affected by cell senescence genes, $\mathrm{p} 16^{\mathrm{INK} 4 \mathrm{a}}$, and cyclin-dependent kinase inhibitors, p21 and p53, the expression of which has been reported to contribute to MSC aging (31). Recent research has shown that in cell senescence, the expansion number is increased within in vitro cultures, and the 3-lineage differentiation ability and proliferation ability of MSCs undergoing in vitro expansion is weakened (19). In our previous study, we found that undifferentiated hUC-MSCs possess certain liver functions (e.g., glycogen storage, LDL uptake, and the expression of ALB and A1AT proteins) (20). However, the question of whether a relationship exists between cell passage and hepatogenic differentiation ability has not yet been examined. Thus, we attempted to study the relationship between cell aging and hepatogenic differentiation efficiency, and to examine why MSCs lose the potential of hepatocyte differentiation.

This study developed a set of experiments to identify cell morphology changes, proliferative rates, gene expression, protein expression, liver function, and the differentiation ability of hUC-MSCs undergoing in vitro aging. Changes in the biological characteristics of the hUC-MSCs revealed that cell senescence was accompanied by changes in cell morphology when the cell passage number increased to P6, with hUC-MSCs losing their typical spindle shape and most cells losing their typical cell morphology at P8. Cell senescence is a complex process, and so we examined the mRNA level of senescence-related genes $\left(\mathrm{p} 16^{\mathrm{INK} 4 \alpha}\right)$ and cyclin-dependent kinase inhibitors (p21 and p53) in hUCMSCs from $\mathrm{P} 4$ to $\mathrm{P} 8$. This revealed that the senescence markers of p53, p16 ${ }^{\mathrm{INK} 4 \alpha}$, and p21 increased with increasing expansion in vitro. Furthermore, flow cytometry analysis indicated that the proliferation ability of these cells gradually decreased at various cell passages. Finally, we investigated the adipogenic and osteogenic potential of hUC-MSC at different passages and found that cells could differentiate into adipocyte-like cells and maintain their osteogenesis ability at both early passage and late passage. We therefore confirmed that P8-hUC-MSCs exhibit senescent properties.

ALB and A1AT are the mature hepatocyte markers in the late stages of liver organogenesis (32). To further confirm that cell aging affects the liver-specific markers and gene expressions of hUC-MSCs, we detected the protein and gene expression of ALB and A1AT in hUC-MSCs at different passages (i.e., from $\mathrm{P} 4$ to P8) using western blot, immunofluorescence staining, and qRT-PCR. Our findings suggest that in vitro senescence leads to gradually decreased mRNA expression levels of liver-specific genes in the undifferentiated hUC-MSCs. At the protein level, the expression of ALB was detected at each passage with no significant difference; however, analysis of the percentage of positive cells in immunofluorescence staining revealed that the expression of A1AT reduced dramatically from $62 \%$ in $\mathrm{P} 4$ to $3 \%$ in $\mathrm{P} 8$. We also examined whether undifferentiated hUC-MSCs possess any liver functions, such as glycogen storage and uptake of LDL and ICG. PAS staining and DiI-acLDL uptake experiments showed that P4-MSCs are better able to store glycogen than P8-MSCs did; however, hUC-MSCs cannot intake ICG regardless of whether or not they have entered the aging state. Thus, undifferentiated hUC-MSCs possess certain liver-specific characteristics that are also affected by cell senescence.

Based on the above results, we confirmed that hUCMSCs lose their biological characteristics and specific liver function at P8. Thus, we used P4-P8-hUC-MSCs to investigate the hepatic differentiation capacity of in vitro-aged hUC-MSCs. In the differentiation process, we used the FH1-based protocol we established in our 
previous study (20) to generate functional hepatocytes from the early and late passages of hUC-MSCs. Liver development mainly experience three dynamic processes: (I) the DE cell formation stage, (II) the liver progenitor specialization stage, and (III) the hepatocyte maturation stage. Thus, the expression of DE markers, hepatocytespecific markers and mature hepatocyte stage markers of differentiated hUC-MSCs from $\mathrm{P} 4$ to $\mathrm{P} 8$ were verified using immunofluorescence staining. SOX17 is a highmobility-group box domain transcription factor and is essential in DE formation (33), while FOXA2 is essential for liver development initiation and possesses DNA-binding domains containing a FOX helix-loop-helix that includes extended loops or winged helix structures (34). Previous studies consider SOX17 and FoxA2 to be the DE markers of hUC-MSCs (35). Our study showed that there was greater DE cell formation in P4-hUC-MSCs than in P8hUC-MSCs.

In the hepatic specification stage, AFP is an marker of early hepatic differentiation of immature fetal hepatocytes (32). HNF4 $\alpha$ is an important transcription factor of the nuclear hormone factor for the functional differentiation of hepatocytes $(36,37)$. The results of protein expression demonstrated that the expression of AFP and HNF4 $\alpha$ was detected in P4-P8-hUC-MSCs-derived hepatic progenitor cells; however, a high level of $\mathrm{AFP}^{+} \mathrm{HNF} 4 \alpha^{+}$ expression was detected in early passage hUC-MSC-derived hepatic progenitor cells. In the hepatocyte maturation stage, we also used immunofluorescence to evaluate the protein expression of ALB and A1AT. Our findings indicate that the percentage of $\mathrm{ALB}^{+} \mathrm{A} \mathrm{AT}^{+}$cell expression decreased markedly from $99.68 \%$ in P4-hUC-MSC-derived HLCs to $7.6 \%$ in P8-hUC-MSC-derived HLCs. The abilities of functional mature hepatocytes were evaluated via PAS staining, urea production assays, ICG intake, LDL uptake, and western blot analysis. The strongest PAS-positive signals and a better ICG and LDL uptake abilities were detected in HLCs derived from P4-P7-hUC-MSCs. These results were similar to the protein expression results for ALB and A1AT of the western blot analysis. Above all, these results indicate that hepatic differentiation efficiency declines in hUC-MSCs undergoing in vitro aging.

Our study revealed there to be a relationship between cell aging and hepatogenic differentiation efficiency; however, clarification of the molecular mechanism of cell aging in HLC production requires further study. Importantly, our findings suggest that $\mathrm{P} 7$ is the optimal passage time for large-scale functional hepatocyte generation from hUC-
MSCs. Our results provide a theoretical basis for the hepatic differentiation of stem cells in vitro.

\section{Conclusions}

Our study showed that hUC-MSCs possess certain characteristics of mature hepatocytes that gradually change following extensive in vitro aging, resulting in a decline in the hepatogenic differentiation efficiency of in vitro-aged hUC-MSCs. Our findings provide insights for future basic science research and clinical applications.

\section{Acknowledgments}

We would like to thank Dr. Zhou Li for his help and valuable suggestions during our study. We thank all members of the laboratory for sharing reagents and advice. Funding: This study was supported by the China Postdoctoral Science Foundation (No. 2016M592375), Hubei Province's Health and Family Planning Scientific Research Project (No. WJ2017Q002), the Wuhan Enterprise Technology Innovation Project (No. 2018060402011244), and the "Guanggu 3551 Talents" Project (1 ${ }^{\text {th }}$ batch; Donghu HighTech Zone, Wuhan, 2018).

\section{Footnote}

Reporting Checklist: The authors have completed the MDAR reporting checklist. Available at https://dx.doi. org/10.21037/atm-21-4918

Data Sharing Statement: Available at https://dx.doi. org/10.21037/atm-21-4918

Conflicts of Interest: All authors have completed the ICMJE uniform disclosure form (available at https://dx.doi. org/10.21037/atm-21-4918). The authors have no conflicts of interest to declare.

Ethical Statement: The authors are accountable for all aspects of the work, including ensuring that any questions related to the accuracy or integrity of any part of the work have been appropriately investigated and resolved.

Open Access Statement: This is an Open Access article distributed in accordance with the Creative Commons Attribution-NonCommercial-NoDerivs 4.0 International License (CC BY-NC-ND 4.0), which permits the non- 
commercial replication and distribution of the article with the strict proviso that no changes or edits are made and the original work is properly cited (including links to both the formal publication through the relevant DOI and the license). See: https://creativecommons.org/licenses/by-nc-nd/4.0/.

\section{References}

1. Asrani SK, Devarbhavi H, Eaton J, et al. Burden of liver diseases in the world. J Hepatol 2019;70:151-71.

2. Kim WR, Lake JR, Smith JM, et al. OPTN/SRTR 2015 Annual Data Report: Liver. Am J Transplant 2017;17 Suppl 1:174-251.

3. Allen JW, Bhatia SN. Improving the next generation of bioartificial liver devices. Semin Cell Dev Biol 2002;13:447-54.

4. Strain AJ, Neuberger JM. A bioartificial liver--state of the art. Science 2002;295:1005-9.

5. Chamuleau RA, Deurholt T, Hoekstra R. Which are the right cells to be used in a bioartificial liver? Metab Brain Dis 2005;20:327-35.

6. Tang X, Li W, Wen X, et al. Transplantation of dental tissue-derived mesenchymal stem cells ameliorates nephritis in lupus mice. Ann Transl Med 2019;7:132.

7. Kestendjieva S, Kyurkchiev D, Tsvetkova G, et al. Characterization of mesenchymal stem cells isolated from the human umbilical cord. Cell Biol Int 2008;32:724-32.

8. Kolf CM, Cho E, Tuan RS. Mesenchymal stromal cells. Biology of adult mesenchymal stem cells: regulation of niche, self-renewal and differentiation. Arthritis Res Ther 2007;9:204.

9. Friedenstein AJ, Gorskaja JF, Kulagina NN. Fibroblast precursors in normal and irradiated mouse hematopoietic organs. Exp Hematol 1976;4:267-74.

10. Yoshimura H, Muneta T, Nimura A, et al. Comparison of rat mesenchymal stem cells derived from bone marrow, synovium, periosteum, adipose tissue, and muscle. Cell Tissue Res 2007;327:449-62.

11. Awad HA, Halvorsen YD, Gimble JM, et al. Effects of transforming growth factor beta1 and dexamethasone on the growth and chondrogenic differentiation of adiposederived stromal cells. Tissue Eng 2003;9:1301-12.

12. Young HE, Mancini ML, Wright RP, et al. Mesenchymal stem cells reside within the connective tissues of many organs. Dev Dyn 1995;202:137-44.

13. Baksh D, Yao R, Tuan RS. Comparison of proliferative and multilineage differentiation potential of human mesenchymal stem cells derived from umbilical cord and bone marrow. Stem Cells 2007;25:1384-92.

14. Hu Y, Liao L, Wang Q, et al. Isolation and identification of mesenchymal stem cells from human fetal pancreas. J Lab Clin Med 2003;141:342-9.

15. Friedenstein AJ, Latzinik NW, Grosheva AG, et al. Marrow microenvironment transfer by heterotopic transplantation of freshly isolated and cultured cells in porous sponges. Exp Hematol 1982;10:217-27.

16. Baxter MA, Wynn RF, Jowitt SN, et al. Study of telomere length reveals rapid aging of human marrow stromal cells following in vitro expansion. Stem Cells 2004;22:675-82.

17. Digirolamo CM, Stokes D, Colter D, et al. Propagation and senescence of human marrow stromal cells in culture: a simple colony-forming assay identifies samples with the greatest potential to propagate and differentiate. $\mathrm{Br} \mathrm{J}$ Haematol 1999;107:275-81.

18. Stolzing A, Jones E, McGonagle D, et al. Age-related changes in human bone marrow-derived mesenchymal stem cells: consequences for cell therapies. Mech Ageing Dev 2008;129:163-73.

19. Yang YK, Ogando CR, Wang See C, et al. Changes in phenotype and differentiation potential of human mesenchymal stem cells aging in vitro. Stem Cell Res Ther 2018;9:131.

20. Luo S, Ai Y, Xiao S, et al. Functional hit 1 (FH1)-based rapid and efficient generation of functional hepatocytes from human mesenchymal stem cells: a novel strategy for hepatic differentiation. Ann Transl Med 2021;9:1087.

21. Dutkowski P, Linecker M, DeOliveira ML, et al. Challenges to liver transplantation and strategies to improve outcomes. Gastroenterology 2015;148:307-23.

22. Dutkowski P, Clavien PA. Solutions to shortage of liver grafts for transplantation. Br J Surg 2014;101:739-41.

23. Hayflick L. Antecedents of cell aging research. Exp Gerontol 1989;24:355-65.

24. Koç ON, Gerson SL, Cooper BW, et al. Rapid hematopoietic recovery after coinfusion of autologousblood stem cells and culture-expanded marrow mesenchymal stem cells in advanced breast cancer patients receiving high-dose chemotherapy. J Clin Oncol 2000;18:307-16.

25. Horwitz EM, Prockop DJ, Fitzpatrick LA, et al. Transplantability and therapeutic effects of bone marrowderived mesenchymal cells in children with osteogenesis imperfecta. Nat Med 1999;5:309-13.

26. Liu WH, Song FQ, Ren LN, et al. The multiple functional roles of mesenchymal stem cells in participating in treating liver diseases. J Cell Mol Med 2015;19:511-20. 
27. Karahuseyinoglu S, Cinar O, Kilic E, et al. Biology of stem cells in human umbilical cord stroma: in situ and in vitro surveys. Stem Cells 2007;25:319-31.

28. Wexler SA, Donaldson C, Denning-Kendall P, et al. Adult bone marrow is a rich source of human mesenchymal 'stem' cells but umbilical cord and mobilized adult blood are not. Br J Haematol 2003;121:368-74.

29. Hayflick L, Moorhead PS. The serial cultivation of human diploid cell strains. Exp Cell Res 1961;25:585-621.

30. Bonab MM, Alimoghaddam K, Talebian F, et al. Aging of mesenchymal stem cell in vitro. BMC Cell Biol 2006;7:14.

31. Shibata KR, Aoyama T, Shima Y, et al. Expression of the p16INK4A gene is associated closely with senescence of human mesenchymal stem cells and is potentially silenced by DNA methylation during in vitro expansion. Stem Cells 2007;25:2371-82.

32. Zhang YN, Lie PC, Wei X. Differentiation of mesenchymal stromal cells derived from umbilical cord Wharton's jelly into hepatocyte-like cells. Cytotherapy

Cite this article as: Luo S, Xiao S, Ai Y, Wang B, Wang Y. Changes in the hepatic differentiation potential of human mesenchymal stem cells aged in vitro. Ann Transl Med 2021;9(21):1628. doi: 10.21037/atm-21-4918
2009;11:548-58.

33. Wang H, Luo X, Yao L, et al. Improvement of cell survival during human pluripotent stem cell definitive endoderm differentiation. Stem Cells Dev 2015;24:2536-46.

34. Kaestner KH. The FoxA factors in organogenesis and differentiation. Curr Opin Genet Dev 2010;20:527-32.

35. An SY, Han J, Lim HJ, et al. Valproic acid promotes differentiation of hepatocyte-like cells from whole human umbilical cord-derived mesenchymal stem cells. Tissue Cell 2014;46:127-35.

36. Watt AJ, Garrison WD, Duncan SA. HNF4: a central regulator of hepatocyte differentiation and function. Hepatology 2003;37:1249-53.

37. Parviz F, Matullo C, Garrison WD, et al. Hepatocyte nuclear factor 4alpha controls the development of a hepatic epithelium and liver morphogenesis. Nat Genet 2003;34:292-6.

(English Language Editor: J. Gray) 